\title{
Modeling and simulation of heat/mass transport, nucleation and growth kinetics in phase transformations
}

\author{
Dmitri V. Alexandrov ${ }^{1, a}$, Peter K. Galenko ${ }^{1,2}$, and Ilya O. Starodumov ${ }^{1}$ \\ ${ }^{1}$ Department of Theoretical and Mathematical Physics, Laboratory of Multi-Scale \\ Mathematical Modeling, Ural Federal University, Lenin ave., 51, Ekaterinburg 620000, \\ Russia \\ ${ }^{2}$ Friedrich-Schiller-Universität-Jena, Physikalisch-Astronomische Fakultät, 07743 Jena, \\ Germany
}

Received 24 August 2019

Published online 11 February 2020

\begin{abstract}
The present theme issue is devoted to recent trends and research directions in the phase transformation phenomena occurring in metastable and heterogeneous materials. All papers are concerned with modern theories, experiments, and computer simulations in the wide area of phase transformations. Particular attention is paid to traditional research domains representing the theoretical background for recent simulations and experiments that are as well specifically highlighted herein. Such rapidly developing research directions as phasefield modeling, laser treatment of surfaces, nanostructures, and influence of external fields on the microstructure formation are specially covered in this issue.
\end{abstract}

\section{Introduction}

It is well-known that phase and structural transformations occurring in metastable and heterogeneous materials attract the attention of researchers working in a broad range of theoretical and applied science varying from materials and condensed matter physics to biophysics and life science (see, among others, [1-17]). This is explained by the fact that such transformations completely determine the concluding state of obtained materials, their structure, and properties. Keeping this in mind, we offer readers the present theme issue that develops recent trends in phase transformation phenomena with special attention to such research areas as crystallization and melting, diffusion, convection and segregation, phase-field modeling, laser treatment and annealing in describing multi-phase and multi-component systems with various applications.

The issue is devoted to theoretical modeling, computer simulations and experimental data of transport processes complicated by nucleation and growth kinetics in phase transformations that occur in condensed matter. Special attention is paid to physical and mathematical models for computer simulation in the analysis of microstructure and properties of materials processed in novel techniques and

\footnotetext{
a e-mail: dmitri.alexandrov@urfu.ru
} 
technologies. First of all, the developing models are devoted to the phase transformations in nano-structural materials, shape-memory materials, and materials obtained under external fields (e.g. electromagnetic fields and forced hydrodynamics flows). Recent trends in developing various approaches to the problems under consideration in materials physics include multi-scale modeling as well as continual models in describing the properties of particulate systems, gradient-stable computational algorithms, high-performance software systems, and complex simulations using high-performance computing clusters.

All manuscripts are grouped into seven sections, namely,

1. Multi-phase and multi-component systems [18-21];

2. Laser treatment and annealing [22-25];

3. Diffusion, convection, segregation [26-32];

4. Nano-structures [33-36];

5. Melting and solidification [37-44];

6. Phase fields [45-48];

7. Applications [49-53].

We hope that this issue will be of interest to a wide range of specialists in materials physics, namely for theoretical physicists and chemists working in an area of condensed and soft matters, for developers of computational algorithms and software programs, for scientists engaged in the field of computational experiments, and, of course, for experimentalists from various fields of materials science. The issue proves to be a convenient forum to discuss modern trends and achievements, find new solutions and strengthen the research collaborations.

In conclusion, we would like to thank all author teams for their remarkable contributions to the present theme issue. We are also grateful to all reviewers for their thorough comments, enabling us to significantly improve the presentation of research works. Finally, we would like to thank the EPJ ST publishing team for useful collaborations in organizing the present theme issue.

This work was supported by the Russian Science Foundation (grant number 16-11-10095).

\section{References}

1. J. Frenkel, Kinetic Theory of Liquids (Dover, New York, 1955)

2. U. Nakaya, Snow Crystals (Harvard University Press, Cambridge MA, 1954)

3. J.W. Mullin, Crystallization (Butterworths, London, 1972)

4. D.V. Alexandrov, A.Yu. Zubarev, Philos. Trans. R. Soc. A 377, 20180353 (2019)

5. G. Bossis, O. Volkova, Y. Grasselli, O. Gueye, Philos. Trans. R. Soc. A 377, 20180211 (2019)

6. D.M. Herlach, P.K. Galenko, D. Holland-Moritz, Metastable Solids from Undercooled Melts (Elsevier, Amsterdam, 2007)

7. D.V. Alexandrov, I.G. Nizovtseva, Philos. Trans. R. Soc. A 377, 20180214 (2019)

8. K.F. Kelton, A.L. Greer, Nucleation in Condensed Matter: Applications in Materials and Biology (Elsevier, Amsterdam, 2008)

9. P.K. Galenko, D.V. Alexandrov, Philos. Trans. R. Soc. A 376, 20170210 (2018)

10. O. Galkin, P.G. Vekilov, J. Am. Chem. Soc. 122, 156 (2000)

11. J. Gao, Philos. Trans. R. Soc. A 376, 20170209 (2018)

12. A. Kao, J. Gao, K. Pericleous, Philos. Trans. R. Soc. A 376, 20170206 (2018)

13. D.A. Barlow, J. Cryst. Growth 470, 8 (2017)

14. V.Ya. Shur, A.R. Akhmatkhanov, Philos. Trans. R. Soc. A 376, 20170204 (2018)

15. A.A. Ivanov, I.V. Alexandrova, D.V. Alexandrov, Philos. Trans. R. Soc. A 377, 20180215 (2019)

16. K. Libbrecht, Snowflakes (Voyageur Press, Minneapolis MN, 2004) 
17. M.M. Abrougui, M.T. Lopez-Lopez, J.D.G. Duran, Philos. Trans. R. Soc. A 377, 20180218 (2019)

18. M.R. Rahul, G. Phanikumar, Eur. Phys. J. Special Topics 229, 145 (2020)

19. E. Kharanzhevskiy, P.K. Galenko, M. Rettenmayr, S. Koch, R. Wonneberger, W. Dreier, D.M. Herlach, M.A. Yagovkina, D.A. Kirilenko, V.A. Bershtein, P.N. Yakushev, L.M. Egorova, V.N. Gurin, M.V. Zamoryanskaya, A.V. Egorov, A.S. Senchenkov, Eur. Phys. J. Special Topics 229, 157 (2020)

20. N. Chtchelkatchev, Eur. Phys. J. Special Topics 229, 167 (2020)

21. N.M. Chtchelkatchev, R.E. Ryltsev, M.V. Magnitskaya, A.A. Rempel, Eur. Phys. J. Special Topics 229, 179 (2020)

22. M.A. Eryomina, S.F. Lomayeva, E.V. Kharanzhevskiy, I.N. Burnyshev, Eur. Phys. J. Special Topics 229, 187 (2020)

23. E.V. Aleksandrovich, K.G. Mikheev, G.M. Mikheev, Eur. Phys. J. Special Topics 229, $197(2020)$

24. G.A. Gordeev, V. Ankudinov, E.V. Kharanzhevskiy, M.D. Krivilyov, Eur. Phys. J. Special Topics 229, 205 (2020)

25. E. Kharanzhevskiy, A. Ipatov, M. Krivilyov, A. Makarov, Eur. Phys. J. Special Topics 229, $217(2020)$

26. V. Golod, L.J. Dobosh, A.E. Kim, Eur. Phys. J. Special Topics 229, 225 (2020)

27. O.V. Kazak, D.V. Alexandrov, P.K. Galenko, Eur. Phys. J. Special Topics 229, 239 (2020)

28. A. Chevrychkina, N. Bessonov, A.L. Korzhenevskii, D. Alexandrov, Eur. Phys. J. Special Topics 229, 253 (2020)

29. H. Gomez, M. Velay-Lizancos, Eur. Phys. J. Special Topics 229, 265 (2020)

30. L.V. Toropova, P.K. Galenko, D.V. Alexandrov, G. Demange, A. Kao, M. Rettenmayr, Eur. Phys. J. Special Topics 229, 275 (2020)

31. G. Buchbinder, P. Galenko, Eur. Phys. J. Special Topics 229, 287 (2020)

32. S. Lomaev, L. Vasil'ev, Eur. Phys. J. Special Topics 229, 295 (2020)

33. A.V. Vakhrushev, A.Yu. Fedotov, Eur. Phys. J. Special Topics 229, 305 (2020)

34. A.F. Abu-Bakr, A.Yu. Zubarev, Eur. Phys. J. Special Topics 229, 315 (2020)

35. A.F. Abu-Bakr, A.Yu. Zubarev, Eur. Phys. J. Special Topics 229, 323 (2020)

36. S. Sureshkumar, S. Muthukumar, M. Muthtamilselvan, D.H. Doh, C.R. Cho, E. Prem, Eur. Phys. J. Special Topics 229, 331 (2020)

37. L. Son, V. Sidorov, G. Rusakov, Eur. Phys. J. Special Topics 229, 347 (2020)

38. P.K. Galenko, D.A. Danilov, Eur. Phys. J. Special Topics 229, 353 (2020)

39. A.A. Ivanov, I.V. Alexandrova, D.V. Alexandrov, Eur. Phys. J. Special Topics 229, 365 (2020)

40. E.V. Makoveeva, D.V. Alexandrov, Eur. Phys. J. Special Topics 229, 375 (2020)

41. D.V. Alexandrov, Eur. Phys. J. Special Topics 229, 383 (2020)

42. I.G. Nizovtseva, Eur. Phys. J. Special Topics 229, 405 (2020)

43. O. Gusakova, V. Shepelevich, D. Alexandrov, I. Starodumov, Eur. Phys. J. Special Topics 229, 417 (2020)

44. A.V. Mokshin, B.N. Galimzyanov, D.T. Yarullin, Eur. Phys. J. Special Topics 229, 427 (2020)

45. I.G. Nizovtseva, N. Moelans, K. Reuther, M. Rettenmayr, D.V. Alexandrov, Eur. Phys. J. Special Topics 229, 433 (2020)

46. A. Salhoumi, D.V. Alexandrov, P.K. Galenko, Eur. Phys. J. Special Topics 229, 439 (2020)

47. A. Subhedar, P.K. Galenko, F. Varnik, Eur. Phys. J. Special Topics 229, 447 (2020)

48. I.G. Nizovtseva, N. Moelans, A.A. Ivanov, Eur. Phys. J. Special Topics 229, 453 (2020)

49. S. Zinchenko, Eur. Phys. J. Special Topics 229, 459 (2020)

50. V.N. Popov, A.N. Cherepanov, Eur. Phys. J. Special Topics 229, 467 (2020)

51. O. Kazak, I. Starodumov, Eur. Phys. J. Special Topics 229, 475 (2020)

52. E. Kondrashov, K. Rusakov, M. Leder, Eur. Phys. J. Special Topics 229, 485 (2020)

53. J. Bazdyreva, V. Belousov, F. Nedopekin, V. Bondarenko, V. Bodriaha, A. Kukharev, Eur. Phys. J. Special Topics 229, 495 (2020) 